\title{
Principales marcos conceptuales aplicados para la evaluación de la salud ambiental mediante indicadores en América Latina y el Caribe
}

\author{
Gabriel Schütz, ${ }^{1}$ Sandra Hacon, ${ }^{1}$ Hilton Silva, ${ }^{2}$ \\ Ana Rosa Moreno Sánchez ${ }^{3}$ y Kakuko Nagatani ${ }^{4}$
}

Forma de citar

Schütz G, Hacon S, Silva H, Moreno Sánchez AR, Nagatani K. Principales marcos conceptuales aplicados para la evaluación de la salud ambiental mediante indicadores en América Latina y el Caribe. Rev Panam Salud Publica. 2008;24(4):276-85.

RESUMEN La construcción de indicadores de salud ambiental para evaluar el efecto adverso de los cambios ambientales sobre el bienestar y la calidad de vida de la población es una meta todavía no alcanzada totalmente en América Latina y el Caribe. Por ello, el Programa de las Naciones Unidas para el Medio Ambiente y la Organización Panamericana de la Salud han convocado a instituciones y especialistas de toda la Región para desarrollar un método integral de evaluación del medio ambiente y la salud. En este trabajo se hace un análisis crítico de varias de las metodologías de evaluación ambiental y sanitaria (integrales o no) y se describen, desde una perspectiva histórica, los marcos conceptuales que fundamentan los principales métodos ordenadores o generadores de indicadores de salud ambiental utilizados en América Latina y el Caribe. Se identificaron dos limitaciones metodológicas recurrentes: a) la fuerte dependencia de datos secundarios, lo que implica la necesidad de una capacidad tecnológica instalada poco accesible en América Latina y el Caribe en la actualidad; y b) la falta de criterios claros para desarrollar instrumentos participativos que faciliten la evaluación de problemas de salud ambiental a nivel local. A pesar de los avances alcanzados en el campo de la salud ambiental en cuanto a la comprensión de su complejidad interdisciplinaria, aún se deben mejorar los mecanismos intersectoriales que favorezcan la discusión e implementación de políticas integradas de medio ambiente y salud.

Palabras clave Salud ambiental, indicadores de salud, indicadores ambientales, América Latina, región del Caribe.

1 Fundación Oswaldo Cruz, Escuela Nacional de Salud Pública, Departamento de Endemias Samuel Pessoa, Río de Janeiro, Brasil. La correspondencia se debe dirigir a Gabriel Schütz, Fundação Oswaldo Cruz, Escola Nacional de Saúde Pública, Rua Leopoldo Bulhões, 1480 Manguinhos, Rio de Janeiro RJ 21041-210, Brazil. Correo electrónico: gabrielschutz@hotmail.com
2 Museo Nacional, Universidad Federal de Río de Janeiro, Río de Janeiro, Brasil.

3 Facultad de Medicina, Universidad Nacional Autónoma de México, Departamento de Salud Pública, México, D.F., México.

4 Programa de las Naciones Unidas para el Medio Ambiente, Oficina Regional para América Latina y el Caribe, Ciudad de Panamá, Panamá.
En 2003, el Programa de las Naciones Unidas para el Medio Ambiente (PNUMA) y la Organización Panamericana de la Salud (OPS), en colaboración técnica con la Fundación Oswaldo Cruz (FIOCRUZ) de Brasil, decidieron 
comenzar a trabajar conjuntamente en el proyecto denominado Global Environmental Outlook (GEO) Salud, una iniciativa creada en respuesta a la Declaración de los Ministros de Medio Ambiente y Salud de las Américas, aprobada en Ottawa, Canadá, en marzo de 2002. En esa ocasión, los Ministros se comprometieron a apoyar programas y estrategias que promuevan un medio ambiente más sano y un mejor estado de salud de la población, dentro de un marco de políticas orientadas a disminuir la inequidad y la pobreza y fomentar formas de desarrollo sostenible. Además, se hizo hincapié en la necesidad de apoyar el desarrollo y el fortalecimiento de capacidades para realizar evaluaciones basadas en indicadores de salud ambiental que faciliten el proceso de toma de decisiones en torno a acciones ambientales y sanitarias integradas a niveles local, nacional y regional.

La finalidad del proyecto GEO Salud es, por lo tanto, crear una dinámica interdisciplinaria, intersectorial y participativa de producción de información científica confiable, dirigida a los responsables de tomar decisiones en los países de América Latina y el Caribe (ALC). Esta dinámica debe contribuir a orientar la implementación de políticas ambientales y sanitarias sostenibles a mediano y largo plazos. La iniciativa de desarrollar un enfoque metodológico generador de indicadores de salud ambiental aplicables en ALC surge por varias razones: primero, porque la mayor parte de las herramientas metodológicas en uso actualmente provienen de experiencias llevadas a cabo en países desarrollados, los que cuentan con una capacidad técnica y científica instalada diferente a la disponible en la mayoría de los países de ALC, especialmente en lo referido al monitoreo de variables ambientales y la disponibilidad de series históricas consolidadas para consulta; segundo, porque la heterogeneidad socioambiental y política de ALC exige la creación de una herramienta de evaluación flexible, capaz de adaptarse a las realidades locales sin perder su capacidad general de comparación; y, finalmente, porque la evaluación inte- gral del medio ambiente y la salud en ALC - una región caracterizada por fuertes desigualdades sociales- no puede excluir el análisis de determinantes socioeconómicas.

En este sentido, el primer paso del proyecto GEO Salud fue discutir la aplicabilidad en ALC de las principales metodologías de evaluación y de salud ambientales ya existentes. ${ }^{5}$ Los criterios utilizados para analizar estas metodologías fueron: a) su capacidad de evaluar mediante indicadores el efecto de los problemas ambientales estudiados sobre la salud; b) su adaptabilidad instrumental a las condiciones presupuestarias y a la capacidad tecnológica instalada en los países de ALC; y c) la incorporación de un enfoque participativo en el procedimiento metodológico.

A continuación se presenta un resumen de la discusión en torno al marco conceptual que caracteriza las metodologías analizadas para el proyecto GEO Salud, seleccionadas por haberse aplicado ya para evaluar — mediante indicadores de salud ambiental- el impacto ambiental sobre la salud de la población en ALC.

\section{PRIMEROS MARCOS ORDENADORES DE INDICADORES AMBIENTALES}

Las evaluaciones ambientales enfrentan la tarea de organizar la información a partir de datos estadísticos que se caracterizan por su naturaleza interdisciplinaria, la variedad de sus temas y fuentes y la diversidad de sus elaboradores y usuarios. En la década de 1980, la búsqueda de un método de organizar la generación y comunicación de información ambiental condujo a la División de Estadísticas de las Naciones Unidas a preparar el conocido esquema para la elaboración de estadísticas del medio ambiente (EEEMA) $(2-4)$.

\footnotetext{
Además de las dos agencias convocantes del proyecto GEO Salud -PNUMA y OPS - y del socio técnico -FIOCRUZ-, en la discusión participaron otras instituciones y especialistas de diversos países latinoamericanos y caribeños (1).
}

Se trata de un marco organizativo que vincula el estado de los componentes del medio ambiente (la flora, la fauna, la atmósfera, el agua, la tierra, los suelos y los asentamientos humanos) con el desarrollo de actividades antrópicas y fenómenos naturales (organizados como categorías de información). Como punto de partida para describir los problemas ambientales, el EEEMA propone dos esquemas básicos, uno enfocado al medio natural (cuadro 1) y otro enfocado a los asentamientos humanos (cuadro 2). Estas categorías de información reflejan el hecho de que los problemas ambientales son consecuencia de las actividades humanas y de fenómenos naturales que afectan al medio ambiente; estos efectos, a su vez, provocan reacciones individuales y sociales tendientes a evitarlos o mitigarlos, lo que establece una secuencia de acción, efecto y reacción.

Dicho de otra manera, este esquema responde a la lógica de organización por componentes del medio ambiente que se incorporan en una secuencia de presión, estado y respuesta. Con esto se sienta un precedente importante para los marcos ordenadores de indicadores que irrumpirán en la década siguiente.

En efecto, los marcos ordenadores surgieron en la década de 1990 como una construcción teórica destinada a organizar los indicadores y presentarlos bajo alguna lógica de interrelación, lo que promovió una interpretación más integral de los mismos. La importancia de los marcos ordenadores reside en su facultad de guiar el proceso de recolección de datos relevantes y convertirlos en instrumentos de comunicación para los usuarios, en tanto ordenan y estructuran la información derivada de distintas fuentes. Al mismo tiempo ayudan a identificar temas para los cuales la información es escasa. Definir un marco ordenador permite elaborar la información para que sus posibles usuarios la puedan utilizar más fácilmente, de ahí que se recomiende que este sea —desde el punto de vista de su utilización- lo menos complejo posible y lo más cercano a las posibilidades y necesidades de cada país (5). 
CUADRO 1. Componentes del esquema para la elaboración de estadísticas del medio ambiente (EEEMA) relacionados con el medio natural

\begin{tabular}{|c|c|c|c|}
\hline $\begin{array}{l}\text { Actividades socioeconómicas y } \\
\text { fenómenos naturales }\end{array}$ & $\begin{array}{l}\text { Efectos ambientales de las } \\
\text { actividades y fenómenos }\end{array}$ & $\begin{array}{l}\text { Reacción ante los efectos } \\
\text { ambientales }\end{array}$ & $\begin{array}{l}\text { Inventarios, existencias y } \\
\text { condiciones básicas }\end{array}$ \\
\hline $\begin{array}{l}\text { Utilización de recursos naturales y } \\
\text { actividades conexas }\end{array}$ & $\begin{array}{l}\text { Agotamiento y aumento de los } \\
\text { recursos }\end{array}$ & $\begin{array}{l}\text { Administración y recuperación de } \\
\text { recursos }\end{array}$ & Recursos biológicos \\
\hline $\begin{array}{l}\text { Emisiones y descargas de } \\
\text { desechos y aplicación de agentes } \\
\text { bioquímicos }\end{array}$ & $\begin{array}{l}\text { Cambios en la calidad del medio } \\
\text { ambiente }\end{array}$ & $\begin{array}{l}\text { Monitoreo y control de la } \\
\text { contaminación }\end{array}$ & Recursos cíclicos y no renovables \\
\hline & & Reacciones del sector privado & Inventarios de ecosistemas \\
\hline \multicolumn{4}{|c|}{$\begin{array}{l}\text { Fuente: Organización de las Naciones Unidas. Un esquema para la elaboración de estadísticas del medio ambiente. Nueva York: Naciones Unidas; 1985. (Informes Estadísticos, serie M, } \\
\text { No. 78.) }\end{array}$} \\
\hline $\begin{array}{l}\text { Actividades socioeconómicas } \\
\text { y fenómenos naturales }\end{array}$ & $\begin{array}{l}\text { Efectos ambientales de las } \\
\text { actividades y fenómenos }\end{array}$ & $\begin{array}{c}\text { Reacción ante los efectos } \\
\text { ambientales }\end{array}$ & $\begin{array}{l}\text { Inventarios, existencias } \\
\text { y condiciones básicas }\end{array}$ \\
\hline $\begin{array}{l}\text { Crecimiento y cambio en los } \\
\text { asentamientos }\end{array}$ & $\begin{array}{l}\text { Cambios en las condiciones de las } \\
\text { edificaciones, la infraestructura y los } \\
\text { servicios }\end{array}$ & $\begin{array}{l}\text { Elaboración de políticas y } \\
\text { programas de asentamientos } \\
\text { humanos }\end{array}$ & $\begin{array}{l}\text { Existencia de edificaciones e } \\
\text { infraestructura }\end{array}$ \\
\hline $\begin{array}{l}\text { Actividades económicas } \\
\text { (agricultura, industria, servicios, } \\
\text { turismo, etc.) }\end{array}$ & Cambios en los recursos vitales & $\begin{array}{l}\text { Monitoreo y control de la } \\
\text { contaminación }\end{array}$ & Inventarios ambientales \\
\hline
\end{tabular}

Fuente: Organización de las Naciones Unidas. Un esquema para la elaboración de estadísticas del medio ambiente. Nueva York: Naciones Unidas; 1985. (Informes Estadísticos, serie M, No. 78.)

De los varios marcos ordenadores existentes en la actualidad, el modelo denominado presión-estado-respuesta (PER) puede considerarse como el precursor. Desarrollado en los primeros años de la década de 1990 por la Organización para la Cooperación y el Desarrollo Económico (OCDE) (6), este modelo surgió como respuesta a la necesidad de los países miembros de contar con la información que les permitiera evaluar el costo ambiental de sus políticas de desarrollo económico.

Conceptualmente, el modelo PER se basa en la premisa de que las actividades humanas ejercen presión sobre el medio ambiente, lo que produce cambios en el estado o las condiciones am- bientales y provoca respuestas sociales a esos cambios, que se manifiestan en la adopción de políticas que afectan al medio ambiente. Ese razonamiento de causalidad directa encuentra su antecedente en el concepto conocido como stress-response, desarrollado en Canadá por Rapport y Friend en 1979 (7). La selección de los indicadores de presión, estado y respuesta se realiza tomando como base tres criterios: la pertinencia política, la precisión del análisis y la mensurabilidad.

El enfoque del modelo PER constituyó un avance al integrar los datos ambientales con los económicos y sociales, no obstante, al hacerlo mediante una concepción lineal no aborda la complejidad multicausal de las interacciones entre los ecosistemas y las acciones antrópicas, así como tampoco aborda de forma explícita los efectos adversos de los problemas ambientales sobre la salud. De cualquier manera, el modelo PER se empleó ampliamente en su momento, no solo en países miembros de la OCDE, sino también en proyectos realizados en países latinoamericanos.

A pesar de sus limitaciones conceptuales, el modelo fue de gran utilidad en el establecimiento de prioridades de intervención, la planificación de políticas, la evaluación de la relación costo-efectividad y la evaluación del desempeño ambiental. Sin embargo, al 
estar dirigido a evaluar apenas presiones actuantes, no permitía generar alertas tempranas que contribuyeran a formular políticas ambientales proactivas. Además, por el hecho de utilizar indicadores de presión muy generales, no se podía especificar la naturaleza de los cambios antrópicos que afectaban a las estructuras y funciones de los ecosistemas en cuestión.

El modelo PER quedó superado cuando las evaluaciones del proyecto GEO del PNUMA incorporaron a las evaluaciones indicadores de impacto ambiental. Se entiende por impacto ambiental cualquier efecto causado por alteraciones del estado del medio ambiente que afecte al ecosistema o la salud de la población humana. Al incorporar indicadores del impacto ambiental sobre la vida social, el modelo llamado presión-estado-impacto-respuesta (PEIR) constituyó un avance conceptual significativo con respecto al PER, cuyas evaluaciones se limitaban a considerar el estado de los recursos ambientales. Por medio del PEIR, el PNUMA ha venido elaborando sus informes ambientales periódicos, en los cuales no solo se traza un panorama del medio ambiente, sino que se evalúan perspectivas (incluidas alertas tempranas). Estos informes se orientan al proceso de toma de decisiones por medio de recomendaciones de acciones ambientales prioritarias, sin embargo, aún no incorporan metodológicamente mecanismos participativos intersectoriales. En América Latina, el modelo PEIR se utilizó inicialmente en evaluaciones urbanas del proyecto GEO Ciudades (8).

\section{INCORPORACIÓN DE INDICADORES DE SALUD A LAS EVALUACIONES AMBIENTALES}

Si bien en el modelo PEIR utilizado en las evaluaciones GEO se suele recurrir a indicadores de salud para caracterizar los cambios ambientales, la problemática de la salud humana como tal se incorporó de lleno a los modelos de evaluación ambiental de la mano de la Organización Mundial de la Salud (OMS) con los

FIGURA 1. Relación entre los componentes del modelo fuerzas impulsoras - presión estado - exposición - efecto - acción (FPEEEA)

Fuerzas impulsoras: crecimiento de la población, proceso de desarrollo económico y tecnológico, intereses políticos y financieros

Presión: perfil del proceso de producción y consumo de bienes y servicios; modalidad de la ocupación del territorio y de la utilización de los servicios de los ecosistemas

Estado: niveles de contaminación o alteración del medio ambiente que de alguna manera amenacen la salud humana; peligros naturales; disponibilidad de recursos naturales; degradación de los servicios del ecosistema

Exposición: exposición externa; vía de ingreso al organismo, dosis de absorción; órganos y sistemas del cuerpo humano afectados

Efecto: sobre el bienestar, la morbilidad

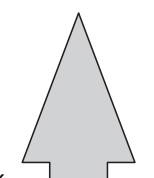
y la mortalidad de la población

Fuente: Corvalán C, Kjellström T. Health and environment analysis for decision making. World Health Stat Q. 1995;48:71-7.

componentes relacionados con la exposición y los efectos en el modelo fuerzas impulsoras-presión-estadoexposición-efecto-acción (FPEEEA). En la figura 1 se muestra la relación entre estos componentes.

El modelo FPEEEA se utilizó en la iniciativa Análisis de Salud y Ambiente para la Toma de Decisiones (HEADLAMP) (9), surgida con el objetivo de contribuir a la formulación de políticas ambientales y de salud mediante el aporte de conocimientos científicamente validados. Esta iniciativa integra métodos de evaluación toxicológica y epidemiológica con métodos de evaluación ambiental a fin de generar y agregar datos en indicadores destinados a monitorear el efecto de los cambios ambientales sobre la salud de la población.

Este proceso integrado se desarrolla en tres etapas consecutivas: la definición del problema, la definición de los indicadores de salud y la formulación y posterior implementación de las intervenciones recomendadas.

El modelo FPEEEA avanza conceptualmente en la construcción de indi- cadores de salud ambiental, ya que asocia la promoción de la salud con el modelo de desarrollo vigente y sus consecuencias ambientales, lo que permite, además, elaborar propuestas intersectoriales de acciones prioritarias integrales. No obstante, debe tenerse en cuenta que tanto la vigilancia epidemiológica como el monitoreo ambiental continuo y sistemático exigen una capacidad tecnológica y de recursos humanos muy alta, lo que dificulta su realización en la totalidad del territorio de todos los países de la Región. De hecho, uno de los mayores problemas que han debido enfrentar ALC para producir estas evaluaciones ha sido la escasez de datos y la sospecha de la baja calidad de muchos de ellos. A pesar de esta limitación, el modelo conceptual FPEEEA se empleó en iniciativas de la OMS en el campo de la salud ambiental, particularmente en la Evaluación de Ecosistemas del Milenio (EEM) y en la determinación de la carga ambiental de enfermedad (CAE).

La EEM es un subproducto de los Objetivos de Desarrollo del Milenio acordados en la Cumbre del Milenio 
de las Naciones Unidas, realizada en 2000 en Nueva York, Estados Unidos de América. En esa cumbre, los 189 Estados Miembros acordaron ocho objetivos, 18 metas y 48 indicadores para medir los progresos hacia el logro de los Objetivos de Desarrollo del Milenio. En 2002, la Conferencia de las Naciones Unidas para el Financiamiento del Desarrollo, llevada a cabo en Monterrey, México, respaldó esos acuerdos. En ese contexto surge la EEM como una herramienta para la evaluación científica de los ecosistemas en todo el mundo, a niveles regional, nacional y local. Este modelo se diseñó para mejorar el manejo de los ecosistemas y su contribución al desarrollo humano mediante la recopilación de la mejor información y los conocimientos disponibles para que sirvan de base a la adopción de decisiones sobre políticas. La EEM parte de la premisa de que los ecosistemas son sistemas complejos dinámicos de comunidades de plantas, animales y microorganismos y su medio ambiente, que interactúan como una unidad funcional y que en su complejidad ofrecen "servicios" a la población humana, es decir, beneficios. Estos beneficios pueden clasificarse en: a) aprovisionamiento, como agua y alimentos; b) regulación, como el control de inundaciones o del clima; c) culturales, como la recreación y el bienestar espiritual; y d) sustentación, como el reciclamiento de nutrientes y la conservación de las condiciones para la vida en el planeta (10).

Indudablemente, la EEM tiene un enfoque integral, pues avanza notablemente en el entendimiento de las relaciones entre el ecosistema y la salud humana, entendida como bienestar. Este modelo permite evaluar amplias dimensiones espacio-temporales $y$ tiene en cuenta complejas interrelaciones. Si se piensa en su aplicación extensa y continua en América Latina, el proceso de construcción de indicadores para la EEM presenta los mismos obstáculos mencionados para los otros modelos, o sea, la escasez de datos de calidad, la incertidumbre en el conocimiento de la estructura y las dinámicas de los ecosistemas, el déficit de capacidad técnica instalada, la falta de recur- sos humanos debidamente capacitados, la inexistencia de series históricas para analizar la evolución de los indicadores, los altos costos y la falta de participación de la comunidad, entre otros. No obstante, la OMS ha venido produciendo numerosos informes de EEM, entre ellos los dedicados al impacto del cambio climático sobre la salud humana.

En cuanto a la CAE - la otra iniciativa de la OMS en el campo de la salud ambiental que utiliza el modelo FPEEEA-, se trata de una adaptación de la determinación de la carga de enfermedad, un procedimiento consolidado en la década de 1990 por Murray y López a partir de indicadores que datan de la década de 1970 (11) y que la OMS ha utilizado para producir informes en los que se estima la "carga" que un determinado riesgo a la salud representa para la sociedad. Esta carga de enfermedad se expresa en años de vida ajustados por discapacidad (AVAD) como resultado de combinar otros dos indicadores: los años de vida perdidos por muerte prematura (AVP) y los años vividos con discapacidad (AVD), ponderados según el grado de discapacidad. Estos indicadores surgen de la aplicación de una serie de operaciones matemáticas bastante complicadas basadas en algunos supuestos, tales como el límite de edad a partir de la cual la muerte ya no es "prematura", lo que repercute directamente en la magnitud de indicadores para riesgos tales como la muerte externa y las enfermedades crónico-degenerativas. Una inconsistencia similar se produce al decidir qué se debe considerar una condición de discapacidad (12).

A fines de la década de 1990 comenzó a aplicarse la CAE para caracterizar los riesgos a la salud resultantes de la exposición a problemas ambientales adversos. La CAE puede servir para: a) medir y comparar la salud de poblaciones o de grupos sociales afectados por problemas ambientales; b) evaluar la evolución de los efectos sobre la salud de estos problemas ambientales; c) medir y comparar la importancia de diferentes factores de riesgo en un momento dado; d) eva- luar los resultados de las intervenciones implementadas; y e) establecer prioridades y orientar la toma de decisiones, la gestión de la salud ambiental y la asignación de recursos (13).

Metodológicamente, la CAE se puede determinar mediante dos enfoques diferentes según el tipo de estudio que se realice. Un enfoque se basa en la exposición y el otro en los escenarios. El primero se aplica cuando se cuenta con datos y medidas de las causas proximales de enfermedad (como los agentes tóxicos o infecciosos) y el segundo se aplica cuando no es posible especificar una relación numérica continua entre las causas proximales de la enfermedad y sus consecuencias. De esta manera, la población estudiada se puede dividir en escenarios de exposición definidos, cada uno de ellos con un riesgo específico (14).

Por ser una herramienta para la planificación de políticas socioambientales que ayuda a determinar las necesidades y los escenarios futuros, la medición de la CAE contribuye con una base racional al proceso de toma de decisiones y a la formulación de políticas. Sin embargo, las estimaciones son muy incompletas, principalmente debido a la falta de datos epidemiológicos, no siempre disponibles a nivel local. Tampoco incorpora aspectos sociales que influyen directamente en el estado de salud de una población, como la inequidad y la pobreza.

Conceptualmente, las estimaciones de carga de enfermedad tienen la dificultad inherente de no considerar a la salud como bienestar, sino como ausencia de enfermedad. En cuanto a las limitaciones metodológicas, no se prevén mecanismos participativos y se requieren procedimientos matemáticos y estadísticos muy complicados que dificultan la apropiación de la información generada.

\section{MÉTODOS PARA LA CONSTRUCCIÓN DE INDICADORES DE SALUD AMBIENTAL}

Desde finales de la década de 1980, en Estados Unidos y Canadá se desa- 
rrollaron nuevos enfoques metodológicos que ayudan a entender — mediante indicadores organizados sobre una base conceptual - la problemática de la salud ambiental, entendida esta como los aspectos de la salud humana que pueden verse afectados por factores ambientales, ya sean químicos, físicos, biológicos, sociales o psicosociales.

Al contemplar la generación de datos primarios, estos métodos permiten superar las dificultades derivadas de la dependencia de datos secundarios, propias de los modelos analizados hasta aquí. A continuación se exponen los principales métodos generadores de información de salud ambiental implementados en ALC, tomando en cuenta tanto el desarrollo teórico como el fortalecimiento de capacidades.

\section{Enfoque ecosistémico en salud humana}

Este método conocido como ECOSALUD, desarrollado por el Centro Internacional de Investigaciones para el Desarrollo (CIID) de Canadá, se propuso promover la salud humana mediante un mejor manejo de los ecosistemas, lo que implica insertar la cultura humana en el medio ambiente en la que surge. Conceptualmente, este enfoque reconoce que la economía, el medio ambiente y las necesidades de la comunidad tienen un impacto determinado en la salud de un ecosistema dado y que, por consiguiente, ese impacto puede afectar la salud de las personas que habitan en dicho ecosistema. Su desafío mayor es, por lo tanto, desarrollar el manejo adecuado del ecosistema mediante el diseño y la ejecución de soluciones intersectoriales en lugar de acciones segmentadas de los sectores salud y ambiental. Para alcanzar sus objetivos, esta iniciativa impulsa la interdisciplinaridad, la equidad de género y el desarrollo de estrategias de comunicación entre los actores sociales, los que participan a lo largo de todo el proceso de investigación, mediante la valorización de los recursos humanos locales y su riqueza cultural (15). Por otro lado, este enfoque asume el gran desafío político de dar respuestas a la comunidad local, a la que se compromete e involucra.

Sin embargo, el procedimiento experimental no deja claro cómo se deben implementar las etapas metodológicas para la generación de los datos. Finalmente, su aplicación exige un profundo conocimiento teórico y empírico de las características del ecosistema, lo que normalmente puede resultar difícil de determinar por más sofisticado que sea el levantamiento que se proyecte implementar. Se debe tener en cuenta que los proyectos de mayor extensión espacio-temporal tienen costos financieros $y$ humanos mucho mayores.

\section{Análisis comparativo de riesgos}

Este es un método creado por la Agencia de Protección Ambiental (EPA) de los Estados Unidos para determinar los riesgos relativos de varios peligros ambientales. Su objetivo general es identificar y atender las áreas de mayor riesgo ambiental, de modo que se establezca un marco de referencia para la definición de las prioridades ambientales en el proceso de toma de decisiones, basado en la ciencia, la política y el análisis económico.

En este método, el riesgo ambiental se clasifica en tres tipos diferentes: riesgo sobre la salud, que a su vez se divide en dos grandes grupos: la exposición a sustancias cancerígenas y la exposición a sustancias no carcinogénicas; riesgo sobre el medio ambiente, cuando hay amenazas a especies individuales, el hábitat, la diversidad de especies y sus interacciones; y riesgo a la calidad de vida, cuando hay amenazas a la estética de un lugar, al bienestar económico o a la igualdad en la distribución de costos y beneficios en una comunidad (tanto en el presente como respecto a generaciones futuras), o cuando se amenaza la tranquilidad, la recreación y la identidad de un grupo, entre otras.

El procedimiento metodológico del análisis comparativo de riesgos consiste en cuantificar el efecto de la exposición a un peligro ambiental sobre la salud de una población y establecer parámetros de comparación con otros factores de riesgo y enfermedad. El método puede esquematizarse en cuatro etapas: la identificación del peligro, la evaluación de la dosis, la evaluación de la exposición y la caracterización del riesgo. El análisis comparativo de riesgos evalúa los diferentes tipos y grados de riesgo a los que está sometida una comunidad debido a un número determinado de problemas ambientales, y con esto ayuda tanto a establecer prioridades como a orientar la asignación de recursos (16).

A lo largo de todo el proceso de evaluación, el análisis comparativo de riesgos busca integrar el análisis técnico-científico a la percepción que la comunidad afectada tiene del riesgo ambiental. De esta forma, este método de análisis constituye una herramienta para la planificación socioambiental que ayuda a determinar las necesidades actuales y futuras, y facilita el proceso de toma de decisiones con la participación social. Sin embargo, este método necesita una gran cantidad de datos toxicológicos y epidemiológicos sin los cuales, las estimaciones producidas resultan muy incompletas. Consecuentemente, son estudios que exigen una gran inversión en términos de recursos financieros, humanos y de infraestructura.

\section{Evaluación de riesgos para la salud pública}

Este método -desarrollado por la Agencia para Sustancias Tóxicas y el Registro de Enfermedades (ATSDR) de los Centros para el Control y la Prevención de Enfermedades (CDC) de los Estados Unidos- no es fácilmente reproducible y presenta algunas limitaciones técnicas importantes, como la falta de dosis de referencia para todos los contaminantes, algo necesario para establecer comparaciones. Finalmente, puede ser un tipo de evaluación demasiado compleja para aplicarse en los servicios de salud, por lo que hasta el momento se ha aplicado muy pocas veces en ALC.

Este modelo, que propone revisar la información disponible sobre las sustancias peligrosas presentes en un sitio dado y analizar si la exposición a esas 
sustancias puede causar algún daño a la población, se emplea con frecuencia en los Estados Unidos para evaluar amenazas ambientales. Entre sus aplicaciones se pueden mencionar: a) exposiciones a productos químicos en áreas donde se libera un contaminante de manera continua en bajas concentraciones, pero que representa un peligro potencial para la salud; b) áreas de pasivos ambientales (es decir, territorios abandonados por la contaminación con substancias tóxicas o radioactivas); y c) en casos de accidentes con productos químicos que comprometen los recursos naturales, su uso y la integridad del ecosistema afectado.

El procedimiento experimental de la evaluación de riesgos de la salud pública consiste en evaluar la exposición, es decir, estimar el daño que las sustancias tóxicas en estudio pudieran causar a las personas. El objetivo es caracterizar la toxicidad de los contaminantes y determinar si vivir o trabajar en las cercanías puede perjudicar la salud de las personas. Para hacer estas determinaciones se debe contar con tres tipos de información: 1) datos del medio ambiente, tales como la información sobre los contaminantes y de cómo las personas pudieran entrar en contacto con ellos; 2) datos de salud, entre ellos la información disponible sobre las tasas de morbilidad y mortalidad en la comunidad y su nivel comparativo con respecto a las tasas estatales (o provinciales) y nacionales; y 3) información sobre las preocupaciones de la comunidad, como informes de la población acerca de cómo el sitio impacta su salud y calidad de vida (17).

Este método constituye una buena herramienta interdisciplinaria para la gestión ambiental, ya que construye un marco sistemático para asignar prioridades a los problemas, asignar recursos y evitar problemas futuros. Además, establece bases científicas para el control de los riesgos.

Sin embargo, este método se basa en un proceso técnico-científico complejo y son pocos los profesionales calificados con las habilidades necesarias para implementar este modelo. Además, no hay un consenso amplio sobre su propósito, su enfoque o sus resulta- dos y no queda claro cómo desarrollar el proceso de participación social. Cabe destacar que se necesitan datos de monitoreo ambiental y de vigilancia epidemiológica para estimar la magnitud del riesgo, datos que no siempre están disponibles en ALC.

\section{Protocolo para evaluar la excelencia de la comunidad en salud ambiental}

Este método, desarrollado por la Asociación Nacional de Funcionarios de Salud de Condados y Ciudades (NACCHO) de los Estados Unidos, también dependiente de los CDC, está diseñado para ayudar a las comunidades a realizar la evaluación del estado de salud ambiental en sus localidades.

La metodología se fundamenta en un proceso investigativo basado en la comunidad para: a) describir y evaluar las condiciones de salud ambiental local y sus problemas; b) identificar la población en riesgo por la exposición a peligros ambientales; c) identificar y recopilar información de importancia relacionada con la salud ambiental; $y$ d) establecer prioridades de acciones locales dirigidas a resolver los problemas de salud ambiental (18).

Al aplicar el protocolo para evaluar la excelencia local en salud ambiental, la comunidad se involucra junto al equipo técnico en el seguimiento de 12 pasos metodológicos para el desarrollo de instrumentos participativos dirigidos a evaluar los problemas locales de salud ambiental. Estos pasos son: a) determinar la capacidad de la comunidad para desarrollar la tarea; b) definir y caracterizar la comunidad; c) organizar un grupo de trabajo en salud ambiental; d) definir las metas, los objetivos y la amplitud de la investigación y la evaluación propuestas; e) generar una lista de los problemas de salud ambiental específicos de la comunidad; f) analizar estos problemas a partir de un sistema de información previamente definido; g) seleccionar los patrones con respecto a los cuales se comparará la situación local; h) crear un perfil detallado de los problemas de salud ambiental; i) organizar los problemas en orden de importan- cia según los intereses; j) definir las prioridades de acción; k) definir un plan de acción; y l) evaluar los progresos y planear acciones futuras.

Esta metodología se ha puesto a prueba con éxito en algunas comunidades de la frontera entre México y los Estados Unidos, pero presenta la gran desventaja de exigir un considerable esfuerzo técnico y una gran inversión en tiempo, recursos humanos y financieros. Si bien tiene la ventaja de poder desarrollarse con datos cualitativos y de no exigir la aplicación de sofisticados métodos de evaluación ambiental o epidemiológica, tiene la desventaja de permitir solamente el control de las situaciones de riesgo, no su prevención. Además, requiere de una planificación estratégica y una infraestructura que no siempre están disponibles en ALC.

Para finalizar, y como anticipo de la discusión, en el cuadro 3 se presenta un resumen de los principales elementos de todos los enfoques metodológicos antes analizados.

\section{DISCUSIÓN}

No existe una herramienta perfecta, por ende, no existe una metodología específica que permita realizar una evaluación integrada del medio ambiente y la salud. El análisis de las metodologías revisadas permite identificar dos limitaciones metodológicas recurrentes:

a) La fuerte dependencia de datos secundarios, lo que implica la necesidad de una capacidad tecnológica instalada poco accesible en América Latina y el Caribe en la actualidad. En general, la disponibilidad de informaciones ambientales cuantitativas (bases de datos consolidadas) es precaria en ALC en estos momentos. Esta precariedad, en principio, tiene varias explicaciones, pero la principal es la coyuntura socioeconómica adversa imperante, que impide dar la prioridad requerida a los problemas ambientales cuando los sectores productivos más relevantes no se 
CUADRO 3. Principales elementos de los modelos de ordenación y generación de indicadores de salud ambiental implementados en América Latina y el Caribe (ALC)

\begin{tabular}{|c|c|c|c|c|c|}
\hline Modelo & $\begin{array}{c}\text { Agencia } \\
\text { (organización/país) }\end{array}$ & $\begin{array}{l}\text { Alcance del } \\
\text { análisis integral }\end{array}$ & $\begin{array}{l}\text { Limitaciones } \\
\text { metodológicas }\end{array}$ & $\begin{array}{l}\text { Mecanismos participativos } \\
\text { en todo el proceso }\end{array}$ & $\begin{array}{l}\text { Dificultades para } \\
\text { su implementación }\end{array}$ \\
\hline $\begin{array}{l}\text { Presión - estado - } \\
\text { respuesta (PER) }\end{array}$ & $\begin{array}{l}\text { Organización para la } \\
\text { Cooperación y el } \\
\text { Desarrollo Econó- } \\
\text { mico, OCDE (Nacio- } \\
\text { nes Unidas) }\end{array}$ & $\begin{array}{l}\text { Integra los aspectos eco- } \\
\text { nómicos, pero no los } \\
\text { sanitarios }\end{array}$ & $\begin{array}{l}\text { Solo usa datos existen- } \\
\text { tes, produce indicadores } \\
\text { muy generales, no esta- } \\
\text { blece previsiones }\end{array}$ & No se consideran & $\begin{array}{l}\text { Se desarrolló para el } \\
\text { contexto de países } \\
\text { desarrollados, aunque } \\
\text { es conceptualmente } \\
\text { simple }\end{array}$ \\
\hline
\end{tabular}

Presión - estado impacto - respuesta (PEIR) PNUMA (Naciones

Fuerzas impulsoras presión - estado exposición - efecto acción (FPEEEA)

Evaluación de ecosistemas del milenio (EEM)

Carga ambiental de enfermedad (CAE)

Enfoques de ecosistemas (ECOSALUD) de riesgos

Evaluación de riesgos para la salud pública

Centro Internaciona de Investigaciones para el Desarrollo, CIID (Canadá)

Agencia de Protección Ambiental, EPA

Agencia para Sustancias Tóxicas y el Registro de Enfer-
Programa de las Naciones Unidas para el Medio Ambiente, Unidas)

Organización Mundial de la Salud, OMS (Naciones Unidas)

Integra los aspectos socioeconómicos, pero no los epidemiológicos

Requiere datos que no siempre están disponibles en ALC o son de calidad dudosa

Integra multisectorialmente los aspectos socioeconómicos, ambientales y sanitarios, pero mantiene el enfoque lineal del modelo biomédico

Organización Mundial de la Salud, OMS (Naciones Unidas)

Escasez de datos de
Es una herramienta para la planificación socioambiental que ayuda a planificar necesidades y escenarios futuros
Las estimaciones suelen ser muy incompletas debido a la falta de datos y consenso respecto a las metodologías de medición; necesita una gran cantidad de datos epidemiológicos (Estados Unidos) medades, ATSDR (Estados Unidos) ciones producidas resul-
Avanza notablemente en el entendimiento conceptual de los factores socioambientales que influyen sobre la salud humana

Permite un buen mapeo de los riesgos ambientales para la salud a los que está sometida una comunidad y facilita la identificación de las prioridades

Aporta un excelente marco sistemático interdisciplinario para asignar prioridades a los problemas, asignar recursos y evitar problemas futuros

Su enfoque integra la percepción de la comunidad y no requiere sofisticados métodos epidemiológicos 0 de monitoreo ambiental calidad, requeridos para la construcción de sus indicadores
Los pasos metodológicos no están claramente definidos; requiere de un nivel de conocimiento del ecosistema no siempre disponible en ALC

Necesita una gran cantidad de datos toxicológicos y epidemiológicos, sin los cuales las estimatan muy incompletas

Necesita un conjunto de datos de monitoreo ambiental y de vigilancia epidemiológica no siempre disponibles o confiables en ALC
Sus pasos metodológicos están bien definidos, pero su aplicación requiere una infraestructura institucional poco frecuente en ALC
Actores sociales participan en la definición de las prioridades, pero no está claro el mecanismo de su participación en el proceso

Actores sociales participan en la definición de las prioridades, pero no está claro el mecanismo de su participación en el proceso

No está clara la influencia de la comunidad en la implementación del proceso

Los actores sociales participan en todo el proceso

No está clara la influencia de la comunidad en la implementación del proceso

No está clara la influencia de la comunidad en la implementación del proceso

Altos costos de la estructuración y la sustentación de las bases de datos y de los sistemas de información exigidos

La vigilancia epidemiológica y el monitoreo sistemático requieren de grandes inversiones y de una infraestructura no siempre disponible en ALC

Los servicios de salud en ALC suelen carecer de los registros de enfermedades no transmisibles que puedan estar asociadas con los cambios ambientales, lo que dificulta la estimación básica por este método

Los costos de su implementación y mantenimiento suelen ser muy altos

Son estudios que exigen una gran inversión en términos de recursos financieros, humanos e infraestructura

Constituye un proceso técnico-científico complejo; en ALC hay pocos profesionales calificados con las habilidades necesarias para implementar este modelo

Es un método participativo a nivel de comunidades que ha mostrado buenos resultados
Exige un considerable esfuerzo técnico y una gran inversión en tiempo, recursos humanos y financieros 
ven afectados. Esto se traduce en la falta de continuidad de los incentivos políticos y financieros, tanto para capacitar recursos humanos como para actualizar adecuadamente la capacidad técnica instalada. En cuanto a los métodos evaluados en los que se parte de la generación de datos primarios, las dificultades observadas son los altos costos y la permanente amenaza de discontinuidad de los programas ambientales, lo que pone en riesgo la posibilidad de consolidar series históricas. Además, estos programas son implementados por agencias internacionales, lo que representa una dificultad para algunos gobiernos reacios a aceptar que terceros, no nacionales, generen indicadores en su soberanía. No obstante, a pesar de la precariedad de la información predominante actualmente en muchos países de ALC, la generación de datos primarios constituye una acción crucial y prioritaria que se debe acometer para poder encarar las demás acciones, es decir, la evaluación, la corrección, el control y la prevención de los efectos adversos sobre la salud provocados por la exposición a factores ambientales.

b) Faltan criterios claros para desarrollar instrumentos participativos que faciliten la evaluación de problemas de salud ambiental a nivel local. Es preciso subrayar que a pesar de los avances alcanzados en el campo de la salud ambiental en cuanto a la comprensión de su complejidad interdisciplinaria, aún se deben mejorar los mecanismos intersectoriales que conduzcan a la discusión e implementación de políticas públicas integradas de medio ambiente y salud. Esta deficiencia repercute tanto en la precisión del enfoque con que se aborda el problema como en el acierto con que se seleccionan las herramientas metodológicas para evaluarlo. La participación de la comunidad, con su sabiduría sobre la problemática local, no solo es importante en la aceptación legitimadora de lo ya decidido por expertos, sino que resulta fundamental en todo el proceso de construcción de la base de información en salud ambiental.

En este sentido, según lo analizado, para avanzar en la construcción de un enfoque integrado de evaluación del medio ambiente y la salud en ALC se recomienda - tal como se propone en el proyecto GEO Salud- pasar por un proceso de maduración conceptual interdisciplinario, de diálogo intersectorial efectivamente participativo, más acorde con la realidad socioeconómica latinoamericana y caribeña. Es en este contexto social que el enfoque metodológico se deberá consolidar, de manera que el avance del conocimiento se base en la interacción dinámica de los diferentes componentes de los ecosistemas locales y los factores determinantes sociales de la salud, en búsqueda de ambientes más sanos y de una mejor calidad de vida.

\section{REFERENCIAS}

1. Programa de las Naciones Unidas para el Medio Ambiente, Organización Panamericana de la Salud, Fundação Oswaldo Cruz. En búsqueda de herramientas y soluciones integrales a los problemas de medio ambiente y salud en América Latina y Caribe. México, D.F.: PNUMA, ORLAC; 2005

2. Organización de las Naciones Unidas. Conceptos y métodos de las estadísticas del medio ambiente: estadísticas de los asentamientos humanos. Informe técnico. Nueva York: Naciones Unidas; 1989. (Estudios de Métodos, serie F, No. 51.)

3. Organización de las Naciones Unidas. Un esquema para la elaboración de estadísticas del medio ambiente. Nueva York: Naciones Unidas; 1985. (Informes Estadísticos, serie M, No. 78.)

4. Organización de las Naciones Unidas. Conceptos y métodos de las estadísticas del medio ambiente: estadísticas del medio natural. Informe técnico. Nueva York: Naciones Unidas; 1992. (Estudios de Métodos, serie F, No. 57.)

5. Quiroga R, Fernández F, Jiles L. Elementos referenciales para la elaboración de estadísticas ambientales en América Latina y el Caribe. Santiago de Chile: CEPAL; 2004.

6. Organization for Economic Co-Operation and Development. Environmental performance reviews: a practical introduction. Paris: OCDE/GD; 1997.

7. Rapport D, Friend A. Towards a comprehensive framework for environmental statistics: a stress-response approach. Ottawa: Statistics Canada; 1979. (Catalogue 11-510.)

8. Programa de las Naciones Unidas para el Medio Ambiente. Metodología para la elaboración de los informes GEO Ciudades. México, D.F.: PNUMA, ORLAC; 2003.

9. Corvalán C, Kjellström T. Health and environment analysis for decision making. World Health Stat Q. 1995;48:71-7.

10. Millennium Ecosystem Assessment. Ecosystems and human well-being. A framework for assessment. Washington, D.C.: Island Press; 2003.

11. Sullivan D. A single index of mortality and morbidity. HSMHA Health Rep. 1971;86(4): 347-54.

12. Pereira Candel J, Cañón Campos J, Álvarez Martín E, Génova Maleras R. La medida de la magnitud de los problemas de salud en el ámbito internacional: los estudios de carga de enfermedad. Rev Admin Sanit. 2001;5(19):441-65.

13. Prüss-Üstün A, Mathers C, Corvalán C, Woodward A. Introduction and methods: assessing the environmental burden of disease at national and local levels. Geneva: World
Health Organization; 2003. (WHO Environmental Burden of Disease Series $\mathrm{N}^{\circ} 1$.)

14. Hacon S, Schütz G, Más Bermejo P. Indicadores de saúde ambiental: uma ferramenta para a gestão integrada de saúde e ambiente. Cad Saude Coletiva. 2005;13(1):45-66.

15. Lebel J. Health: an ecosystem approach. Montreal: IDRC; 2003.

16. US Environmental Protection Agency. Framework for ecological risk assessment. Risk Assessment Forum. Washington, D.C.: US EPA; 1992.

17. Agency for Toxic Substances and Disease Registry. Public health assessment guidance manual. Atlanta: ATSDR; 2002.

18. National Association of County and City Health Officials. Protocol for assessing community excellence in environmental health: a guidebook for local health officials. Washington, D.C.: NACCHO; 1992.

Manuscrito recibido el 12 de abril de 2007. Aceptado para publicación, tras revisión, el 22 de mayo de 2008. 
ABSTRACT The establishment of environmental health indicators for assessing the adverse effects of environmental changes on the population's health and quality of life is, as yet, a goal that has not been fully reached in Latin America and the Caribbean. As such, the

\section{Application of key frameworks to an indicator-based evaluation of environmental health in Latin America and the Caribbean}

Key words Environmental health, health indicators, environmental indicators, Latin America,
United Nations Environment Program and the Pan American Health Organization (PAHO) have convened Region's institutions and experts to develop a comprehensive method for assessing environmental health. This paper evaluates several methodologies, comprehensive or otherwise, for assessing health and the environment, and describes the frameworks that have historically undergirded the key methods that have either structured or generated the environmental health indicators being used in Latin America and the Caribbean. The recurring, methodological limitations were identified: (a) relying heavily on secondary data, which points out the need for technological infrastructure that is rarely available in Latin America and the Caribbean today; discussion of environmental health issues at the grass-roots level. Despite the progress made by the field of environmental health with regard to understanding its interdisciplinary complexities, intersectoral operations must be improved to favor open communication and implementation of integrated policies on environmental and health.

\section{Caribbean Region.} and (b) a lack of clear criteria for developing inclusive tools that would facilitate the

\begin{tabular}{|c|c|}
\hline $\begin{array}{l}2007 \text { • } 56 \text { pp. } \\
\text { ISBN } 9789243563169 \\
\text { Código: WHO } 65 \\
\text { Precio: US\$10.50 en } \\
\text { América Latina y el } \\
\text { Caribe/ US\$ } 15.00 \text { en el } \\
\text { resto del mundo }\end{array}$ & $\begin{array}{l}\text { doméstica y salud. Combustibles para una vida mejor } \\
\text { El uso de energía es fundamental para satisfacer nuestras necesidades más bási- } \\
\text { cas: cocinar, hervir agua, alumbrarnos y calentarnos. Más de } 3 \text { mil millones de per- } \\
\text { sonas siguen quemando madera, estiércol, carbón y otros combustibles tradicionales } \\
\text { en sus hogares. La contaminación del aire en interiores causa más de 1,5 millones } \\
\text { de defunciones al año, principalmente entre niños pequeños y madres. Además, ese } \\
\text { tipo de contaminación y el uso ineficiente de la energía en los hogares representan } \\
\text { un obstáculo en nuestro camino para alcanzar los Objetivos de Desarrollo del Mile- } \\
\text { nio. En este libro se resume el impacto de la energía doméstica en la salud y se pre- } \\
\text { senta a grandes rasgos la evolución de la utilización de combustibles sólidos y las } \\
\text { tendencias futuras en ese sentido. Se analizan también diversas intervenciones y se } \\
\text { demuestra, cuantificando los costos y los beneficios de algunas de ellas, que existen } \\
\text { soluciones que representan una buena inversión. } \\
\text { Envíe su pedido y pago en dólares estadounidenses a: http://publications.paho.org; } \\
\text { correo electrónico: paho@brightkey.net; Fax: (301) 209-9789; } \\
\text { Tel: (301) 617-7806; (1-800) 472-3046 (U.S. solamente) }\end{array}$ \\
\hline
\end{tabular}

